\title{
Reduction of nonlinear patterning effects in SOA-based All-optical Switches using Optical filtering
}

Nielsen, Mads Lønstrup; Mørk, Jesper; Skaguchi, J.; Suzuki, R.; Ueno, Y.

Published in:

Technical Digest OFC 2005

Publication date:

2005

Document Version

Publisher's PDF, also known as Version of record

Link back to DTU Orbit

Citation (APA):

Nielsen, M. L., Mørk, J., Skaguchi, J., Suzuki, R., \& Ueno, Y. (2005). Reduction of nonlinear patterning effects in SOA-based All-optical Switches using Optical filtering. In Technical Digest OFC 2005 (Vol. Paper OThE7). IEEE.

\section{General rights}

Copyright and moral rights for the publications made accessible in the public portal are retained by the authors and/or other copyright owners and it is a condition of accessing publications that users recognise and abide by the legal requirements associated with these rights.

- Users may download and print one copy of any publication from the public portal for the purpose of private study or research.

- You may not further distribute the material or use it for any profit-making activity or commercial gain

- You may freely distribute the URL identifying the publication in the public portal 


\title{
Reduction of Nonlinear Patterning Effects in SOA-based All- Optical Switches using Optical Filtering
}

\author{
Mads L. Nielsen and Jesper Mark \\ Research Center COM, Technical University of Denmark, Building 345V. DK-2800 Kgs. Lyngby, Denmark (minacom.dtudk) \\ Jun Sakaguchi, Rei Suzuki, and Yoshiyasu Ueno \\ Gractuate School of Electronic Engineering, University of Electro-Communications, 1-5-1 Chofugaoka, Chofu, Tokyo, 182-8585, Japan
}

\begin{abstract}
We explain theoretically, and demonstrate and quantify experimentally, how appropriate filtering can reduce the dominant nonlinear patterning effect, which limits the performance of differential-mode SOA-based switches.

(C)2005 Optical Society of America

OCIS codes: (060.4510) Optical communications; (070.4340) Nonlinear signal processing; (250.5980) Semiconductor optical amplifiers; (190.2620) Frequency conversion; (120.2440) Filters.
\end{abstract}

\section{Introduction}

Semiconductor optical amplifier (SOA) based all-optical switches are attractive candidates for realizing essential subsystems for optical networks, such as wavelength converters, regenerators, and optical time-division demultiplexers [1]. In terms of footprint and power consumption, an SOA is superior as a nonlinear medium in an all-optical switch, compared to e.g. a nonlinear fiber. However, the operating speed is limited by the recovery of the carrier density, which takes place on a time scale of typically $20-100 \mathrm{ps}$, and leads to patterning effects on the switched signal, which become detrimental at high bitrates. This problem has been addressed by operating SOAbased interferometric switches in the so-called differential mode (DM) [2], which effectively cancels the long recovery of the carrier density induced gain and phase changes. This approach creates a short switching window, and eliminates the contribution to the patterning effect from the slow recovery of the switched power, which will be referred to as the linear patterning effect [3]. However, in the absence of linear patterning, another patterning effect - nonlinear patterning (NLP) - will dominate [3]. This effect can be attributed to gain saturation and manifests itself as a fluctuation of the switched pulse power.

In this paper we analyze the patterning induced by a Delayed Interference Signal Converter (DISC), consisting of an SOA and an Asymmetric Mach-Zehnder Interferometer (AMZI). We explain how detuning an optical filter at the output of the DISC reduces the NLP - an effect also noted in [4] - and show that it constitutes a general way of reducing NLP induced by DM switches. The improvement is quantified through simulations as well as experiments.

\section{Principle and modeling}

The DISC is illustrated in Fig. 1 (a) with an AMZI including a differential delay of $\tau$ and a phase offset of $\Phi_{0}$. Fig. 1 (b) shows a modeling result of the phase modulation of the CW probe at the output of the two branches of the AMZI for a $40 \mathrm{~Gb} / \mathrm{s} \mathrm{RZ}$ input data signal pattern "11011100". Considering the phase modulation in e.g. the lower arm, $\Phi_{\mathrm{N}}(\mathrm{t})$, it is observed to only partially recover during the timeslot containing the first logic " 0 ". Thus, if this phase modulation is generated in one arm of a so-called standard-mode interferometric switch [5], where only one of the two interfering signals is modulated, the extinction ratio of the switched probe will be very small.

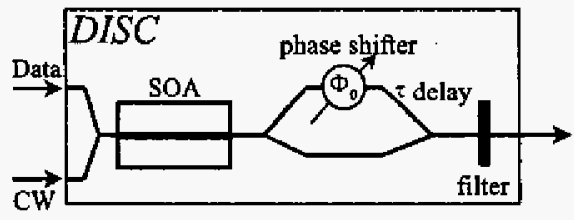

(a)

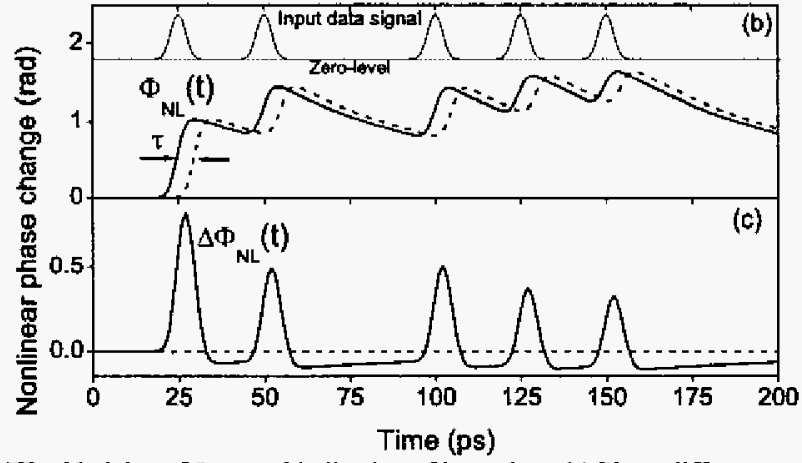

Fig. 1. (a) Schematic of the DISC. (b) Probe phase in arms of AMZI with delay of 5 ps, and indication of input data. (c) Phase difference.

However, the transmittance of an interferometer is governed by the phase difference between its arms, and as illustrated in Fig. 1 (c) the phase difference $\Delta \Phi_{\mathrm{N} L}(\mathrm{t})=\Phi_{\mathrm{NL}}(\mathrm{t})-\Phi_{\mathrm{NL}}(\mathrm{t}-\tau)$ opens a switching window defined by the 


\section{OThE7}

delay $\tau$, which allows for operation at significantly higher bitrates compared to the standard-mode interferometric switch. Fig. 1 (c) shows that the DISC eliminates the patterning effect stemming from the slow recovery of the phase response. Since this effect can be attributed to the linear part of the carrier rate equation, we refer to it as linear patterning [3]. However, it is clear from Fig. 1 (c) that the magnitude of the phase difference $\Delta \Phi_{\mathrm{NL}}(\mathrm{t})$, introduced by the input data pulses, depends on the data pattern. This is because the gain available to a specific data pulse - and thus the magnitude of the phase difference $\Delta \Phi_{\mathrm{NL}}(t)$ - depends on the level of saturation. Since this effect can be attributed to the nonlinear term of the carrier rate equation, we refer to it as noninear patterning [3].

The fact that the phase shift that may be induced by a specific data pulse depends on the available gain can be exploited to limit the NLP. This is because the larger the available gain, the larger is the chirp that is introduced on the switched probe signal. If two closely spaced data pulses are launched into an SOA along with a CW probe, the trailing pulse chirps the probe less than the leading pulse. Passing the probe through an AMZI, as in the DISC configuration, does not change this fact. Consequently, by suppressing the highly chirped parts of the signal compared to the less chirped parts, by filtering at the DISC output, the transmittance of the switched pulse with highest chirp may be reduced relatively to the trailing pulse with the lower chirp, thereby reducing the level of NLP. It should be noted that this effect is not limited to the DISC, but may be applied generally to DM switches.
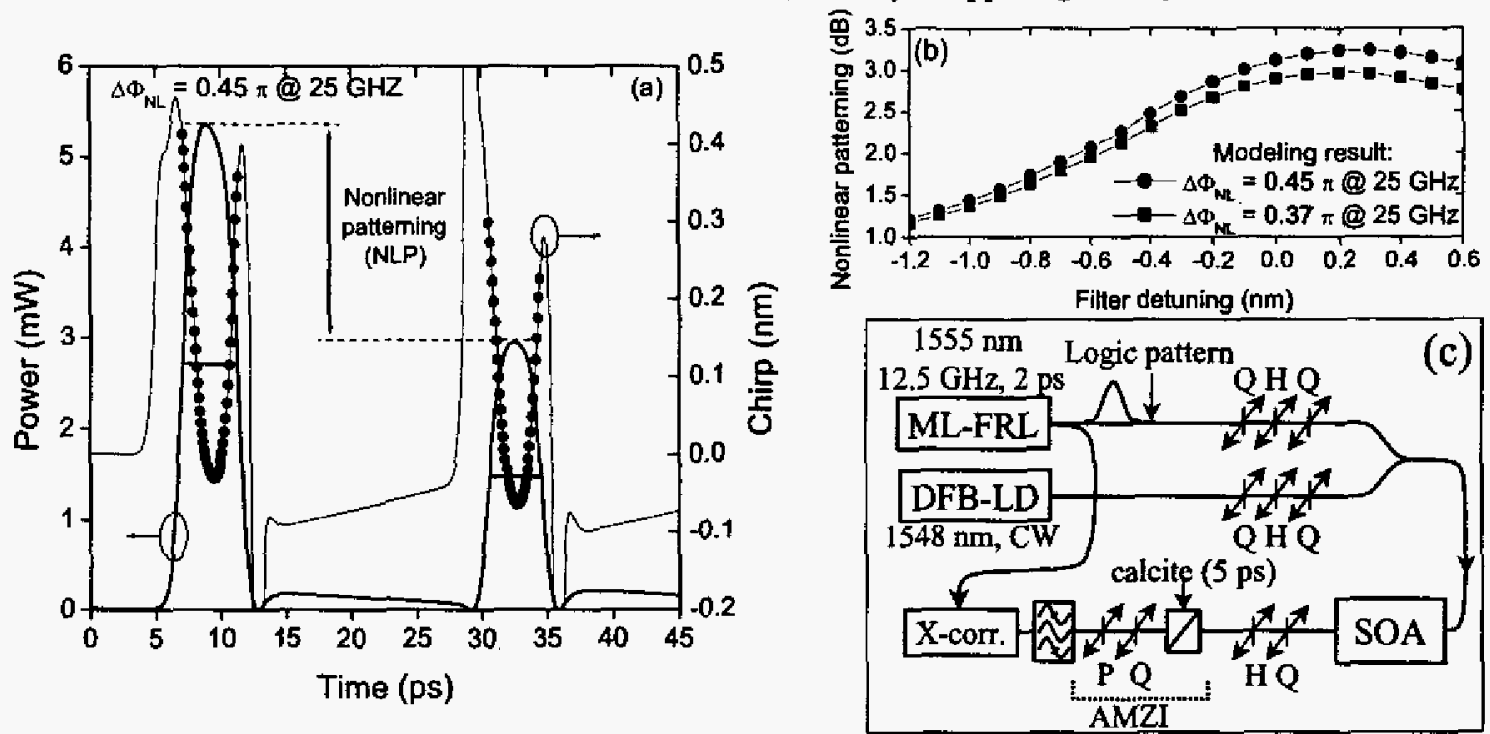

Fig. 2. Simulated DISC output (power and chirp). Horizontal double-arrows indicate FWHM (b) Simulated nonlinear patterning. (c) Experimental setup. Q: quarter-wave plate, $\mathrm{H}$ : balf-wave plate, P: polarizer.

The chirp properties are illustrated in Fig. 2 (a), which shows a simulation of the switched probe power and chirp at the output of the DISC, using a detailed SOA-model including Amplified Spontaneous Emission (ASE). The CW input power to the SOA is $+9 \mathrm{dBm}$, the small-signal gain at the probe wavelength is $30 \mathrm{~dB}$, and the energy of the 2 ps wide (FWHM) Gaussian data pulses are adjusted to provide a nonlinear phase shift of $0.45 \pi$ when the data signal consists of a $25 \mathrm{GHz}$ clock signal. This is a convenient way of stating the level of saturation, since the phase shift induced by a train of short pulses can be estimated from experimental probe spectra, as detailed in [2]. The input data signal used in Fig. 2 (a) is "00110011..." with a bitrate of $43 \mathrm{~Gb} / \mathrm{s}$, the differential delay in the DISC is $\tau=5 \mathrm{ps}$, and the phase offset is $\Phi_{0}=0.98 \pi$. In the following the NLP will be quantified as the peak power ratio of the first to the second pulse, as indicated in Fig. 2 (a). This quantity clearly depends on the number of succeeding pulses [as illustrated in Fig. 1 (c)], as well as the bitrate, and considering just two pulses thus slightly underestimates the peak power fluctuations that would be observed for longer pattern lengths.

The chirp of the switched signal is shown on the right axis of Fig. 2 (a), and a number of distinct features are observed: first, the leading and trailing edges of the switched pulses are strongly red-shifted due to carrier depletion by the data input signal, whereas a slight blue-shift is observed around the pulse peaks owing to carrier recovery. Secondly, the chirp grows to a numerically very high value when the switched pulse power goes to zero (time $=$ $12.7,29.2$, and $35.8 \mathrm{ps})$. This behavior is related to the sign change of the phase difference $\Phi_{\mathrm{NL}}(t)-\Phi_{\mathrm{NL}}(\mathrm{t}-\tau)-\Phi_{0}$, which also explains the generation of the "satellite pulse" after each switched pulse, as detailed in [6]. Finally, the red-shift across the FWHM of the switched pulses, indicated by the double-arrows in Fig. 2 (a), is larger for the leading pulse compared to the trailing pulse, due to the difference in available gain (carrier density). This is emphasized by the black dots on the chirp curve in the time spans corresponding to the FWHM of the switched pulses. 


\section{OThE7}

Passing the DISC output though a BPF with the peak transmittance tuned to the blue side of the CW wavelength, the NLP may be reduced significantly. This is illustrated in Fig. 2 (b), which shows a simulation of the NLP as a function of the detuning of a $1 \mathrm{~nm}$ wide Gaussian BPF for pulse energies corresponding to nonlinear phase shifts of $0.37 \pi$ and $0.45 \pi$ at a clock frequency of $25 \mathrm{GHz}$. The reduction of NLP by detuning the filter by $-1.2 \mathrm{~nm}$ is observed to be 1.5-2 dB. Moreover, the level of patterning increases with the pulse energy (larger for $\Delta \Phi_{\mathrm{NL}}=0.45 \pi$ compared to $\Delta \Phi_{\mathrm{NL}}=0.37 \pi$ ), which is because more energetic pulses saturate the SOA more, enhancing the difference in gain available to the two pulses. As expected, the difference between the two curves in Fig. 2 (b) decreases for blue detunings, since the associated chirp difference between the switched pulses has an equalizing effect on the peak pulse powers as explained above.

\section{Experimental results}

The experimental setup is shown in Fig. 2 (c): a $12.5 \mathrm{GHz}$ train of 2 ps wide clock pulses at $1555 \mathrm{~nm}$ is emitted from a mode-locked fiber ring laser (ML-FRL), and a data pattern " $00110011 \ldots$ " is created by applying a delay of 23.25 ps $(1 /(43 \mathrm{GHz}))$ in a 1:2 passive fiber-based interleaver. This is launched into an SOA along with a $\mathrm{CW}$ beam at $1548 \mathrm{~nm}$. The polarization states of both signals are aligned to TE using the combination of quarter-wave $(Q)$ and half-wave $(\mathrm{H})$ plates. At the output of the SOA the signals enter an AMZI realized by a calcite crystal with a 5 ps delay between the main axes, and a quarter-wave plate and polarizer (P) for adjusting the phase offset $\Phi_{0}$. At the input of the AMZI, wave plates ensure that the cross-phase modulated probe signal enters the calcite linearly polarized at an angle of $45^{\circ}$ between the main axes. After the DISC the probe signal is separated from the data by a $1 \mathrm{~nm}$ wide (FWHM) Gaussian BPF, before the probe is analyzed using a cross-correlator. Fig. 3 (a) [left axis] shows the measured NLP vs. filter detuning for nonlinear phase shifts at $25 \mathrm{GHz}$ of $0.37 \pi$ and $0.45 \pi$. The results verify the trends predicted by the simulations, although the simulations overestimate the NLP somewhat. The right axis of Fig. 3 (a) shows the average switched power after the BPF, illustrating that there is a trade-off between the reduction of NLP and output power. The reduction of the output power translates into an OSNR reduction. In Fig. 3 (b), crosscorrelation traces for zero detuning and a detuning of $-0.84 \mathrm{~nm}$ are compared for $\Delta \Phi_{\mathrm{NL}}=0.37 \pi$ at $25 \mathrm{GHz}$, and the reduction of NLP is clearly observed.
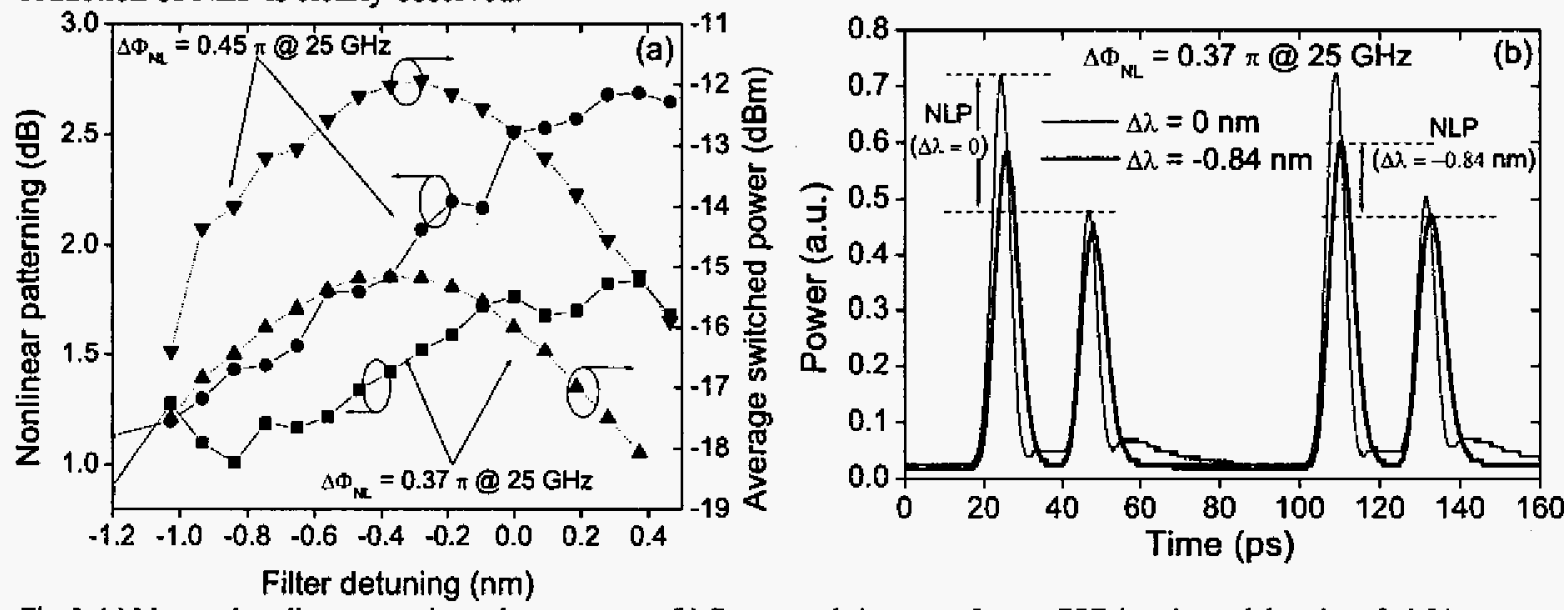

Fig. 3. (a) Measured nonlinear patterning and output power. (b) Cross-correlation traces for zero BPF deturing and deruning of $-0.84 \mathrm{~nm}$.

\section{Summary}

We have demonstrated that the nonlinear patterning effect, which manifests as pulse peak power fluctuations of signals switched by SOA-based differential-mode interferometric switches, can be reduced by the enhancement of blue-shifted spectral components compared to red-shifted. This was realized by detuning a band pass filter towards the blue side of the spectrum, reducing the nonlinear patterning from $2.5 \mathrm{~dB}$ to $1,1 \mathrm{~dB}$. The effect is very general and can be applied to any SOA-based differential-mode switch.

\section{References}

[1] A. Mecozzi et al., "The roles of semiconductor optical amplifiers in optical networks", OPN, March 2001, pp. 36-42, 2001

[2] Y. Ueno et al., "Nonlinear phase shifts induced by SOAs with control pulses ...", JOSA B, 19 (11), pp. 2573-2589, 2002

[3] M. L. Nielsen et al., 'Increasing the modulation bandwidth of SOA based switches using optical filtering", JOSA B, 21 (9), pp. 1606-19, 2004

[4] J. Leuthold et al,, "All-optical wavelength conversion between 10 and $100 \mathrm{~Gb} / \mathrm{s} . . . "$, Opt. Quantum Electron., 33, pp. 939-952, 2001

[5] B. Dagens et al, "New modular SOA-based active-passive integrated Mach-Zehnder interferometer ...", in Proc. ECOC 2002, PD3.1

[6] Y. Ueno et al., "Theoretically predicted nonlinear phase imbalance requirement...", Jpn. J. Appl. Phys., 43 (5B), pp. L665-L668, 2004 